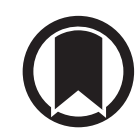

CrossMark

\title{
The MUC5B promoter risk allele for idiopathic pulmonary fibrosis predisposes to asbestosis
}

\author{
To the Editor:
}

Asbestosis is a fibrosing interstitial pneumonitis, which can develop following occupational asbestos exposure and a lengthy latency period [1]. Even after strict regulations on the asbestos industry, an estimated 3400 individuals worldwide died as a consequence of asbestosis in 2017 [2].

Asbestosis shows several similarities with idiopathic pulmonary fibrosis (IPF), with both patient groups consisting of predominantly older, former smoking males [3]. Furthermore, it has been reported that patients with end-stage asbestosis can demonstrate a usual interstitial pneumonia (UIP) pattern on high-resolution computed tomography (HRCT), a pattern that is characteristically diagnostic for IPF if known causes of interstitial lung disease (ILD) are excluded [4].

IPF is more thoroughly investigated than asbestosis and several genetic risk factors are implicated in the pathogenesis of IPF. One of the strongest genetic risk factors is a single nucleotide polymorphism (SNP) located in the promoter region of MUC5B (rs35705950). SEIBOLD et al. [5] demonstrated that, for IPF, the odds ratio (OR) for individuals who were heterozygous for this SNP is 9.0 (95\% CI 6.2-13.1). In the current study, a possible association between the minor allele of MUC5B (rs35705950) and asbestosis was investigated in multiple cohorts.

Patients were included between April 2014 and December 2017. Asbestosis is defined according to American Thoracic Society guidelines [1] and includes thorough occupational history, appropriate latency period and HRCT findings. Three cohorts of patients with asbestosis were included: a discovery cohort from the St Antonius Hospital Nieuwegein, the Netherlands $(n=29)$; replication cohort I recruited via the Institute for Asbestos Victims located in The Hague, the Netherlands $(n=28)$; and replication cohort II consisting of 21 patients from the Bichat-Claude Bernard Hospital Paris, France. Furthermore, a pleural plaque cohort $(n=25)$ without evidence of interstitial fibrosis was included as a reference population for genotype analysis.

Healthy, white, unrelated Dutch $(n=300)$ and French subjects $(n=300)$ were included for comparison with the Dutch and French patients, respectively. All subjects provided written informed consent and ethical approval was received for this study (Dutch: R-05.08A; French: CPP 110104 and 0811760). The MUC5B rs35705950 polymorphism was genotyped using a pre-designed Taqman SNP genotyping assay (Applied Biosystems, Foster City, CA, USA) on DNA isolated from whole blood. Our smallest patient cohort consisted of 21 individuals. Based on the reported minor allele frequencies (MAFs) of $37.5 \%$ in IPF and $9.1 \%$ in healthy subjects [5] and the use of 300 healthy controls, we obtained a power over $80 \%$ in this and our other cohorts.

In total, 78 patients with asbestosis were included with male predominance (97.4\%) and median age of 72.2 years. The mean \pm SD forced vital capacity (FVC) was $81.8 \pm 20.4 \%$ of predicted and diffusion capacity of the lung for carbon monoxide $\left(D_{\mathrm{LCO}}\right)$ corrected for haemoglobin level $52.8 \pm 14.6 \%$ of predicted. There were $67(85.9 \%)$ former or current smokers with an average smoking history of $21.4 \pm 14.5$ pack years. The majority of patients (90\%) reported prolonged occupational exposure to asbestos and all patients demonstrated pleural and/or diaphragmatic plaques on HRCT. A UIP pattern was present on HRCT in 36 patients (46.2\%), a probable UIP pattern in 20 patients $(25.6 \%)$, indeterminate UIP pattern in 16 patients (20.5\%), and a pattern suggestive of an alternative diagnosis in six patients (7.7\%). No lung biopsies or lung transplantations were performed.

@ERSpublications

The minor allele of MUC5B rs35705950 predisposes to asbestosis, but has no effect on survival http:// bit.ly/382gPBM

Cite this article as: Platenburg MGJP, Wiertz IA, van der Vis JJ, et al. The MUC5B promoter risk allele for idiopathic pulmonary fibrosis predisposes to asbestosis. Eur Respir J 2020; 55: 1902361 [https://doi.org/ 10.1183/13993003.02361-2019]. 
For each cohort, the MUC5B rs35705950 SNP was in Hardy-Weinberg equilibrium. MAFs in the discovery, replication I and replication II cohorts were $33 \%, 27 \%$ and $29 \%$, respectively. The minor allele of rs35705950 showed significant association with asbestosis in the discovery cohort, replication I cohort and replication II cohort when compared with healthy controls. Pooled analysis demonstrated that the MAF in patients with asbestosis was $29 \%$ compared to $11 \%$ in healthy controls (table 1 ). MAF in patients with pleural plaques was $14 \%$ and significantly different from patients with asbestosis (OR 2.6, 95\% CI $1.1-6.1 ; \mathrm{p}=2.9 \times 10^{-2}$ ), but did not differ from controls.

A total of 31 patients (39.7\%) died during follow-up. Deaths were attributed to progression of pulmonary fibrosis (64.5\%), lung cancer (3.2\%), non-respiratory (22.6\%) or unknown causes $(9.7 \%)$. Mean survival after diagnosis was 1.9, 3.6 and 5.4 years for the discovery, replication I and replication II cohorts, respectively. FVC and $D_{\mathrm{LCO}}$ tended to be lower in patients from the St Antonius Hospital compared to the other cohorts, but this was not significant. We speculate that patients from the St Antonius Hospital are diagnosed at a later stage compared to patients diagnosed in other Dutch hospitals, as the St Antonius Hospital is a tertiary referral centre. In addition, we hypothesise that the longer survival of the French asbestosis cohort is due to shorter diagnostic delay as a result of nationwide screening programmes of French asbestos exposed workers [6].

In the pooled asbestosis cohort, no differences were detected between baseline characteristics of carriers $(\mathrm{n}=42)$ and non-carriers $(\mathrm{n}=36)$ of the MUC5B risk allele. Carriership of the minor allele of MUC5B rs35705950 could not be identified as a predictor for death in the asbestosis cohort. However, replication in a larger cohort is required to draw any definite conclusion.

In this study, we demonstrated that the MUC5B rs35705950 minor allele predisposes to asbestosis. Previous studies showed that the MUC5B rs35705950 polymorphism is associated with other forms of pulmonary fibrosis, such as IPF, idiopathic non-specific pneumonitis and rheumatoid arthritis-associated ILD $[5,7,8]$. Our study complements these findings and point towards shared pathogenic processes between asbestosis and progressive fibrosing diseases such as IPF. There is evidence that chronic endoplasmic reticulum stress and alveolar epithelial cell apoptosis, pathological processes believed to be involved in IPF pathogenesis [9], also play a role in the aetiology of asbestosis. KAMP et al. [10] demonstrated that alveolar type II cells of rats exposed to asbestos fibres upregulate proteins linked to endoplasmic reticulum stress. Moreover, they also reported that asbestos created a two-fold increase in apoptosis in human alveolar epithelial cells (A549 cells) compared with control experiments.

A minority of subjects with inhalation exposure to asbestos develop asbestosis, however, many develop pleural plaques. A lack of association was found between the cohort of only pleural plaques patients and MUC5B rs35705950. This is in congruence with the general perception that development of pleural plaques does not necessarily lead to other pulmonary diseases.

Our study highlights commonalities between IPF and asbestosis. Nevertheless, both diseases are treated differently. In IPF, it was shown that immunosuppression is harmful $[11,12]$ and that antifibrotic

TABLE 1 MUC5B rs35705950 genotype, minor allele frequency, and odds ratio in patients with asbestosis

\begin{tabular}{|c|c|c|c|c|c|c|}
\hline $\begin{array}{l}\text { Pooled } \\
\text { cohort }\end{array}$ & $\begin{array}{l}\text { Discovery } \\
\text { cohort }\end{array}$ & $\begin{array}{l}\text { Replication } \\
\text { cohort I }\end{array}$ & $\begin{array}{l}\text { Replication } \\
\text { cohort II }\end{array}$ & $\begin{array}{l}\text { Pleural } \\
\text { plaques } \\
\text { without } \\
\text { asbestosis }\end{array}$ & $\begin{array}{l}\text { Healthy } \\
\text { controls } \\
\text { I }\end{array}$ & $\begin{array}{l}\text { Healthy } \\
\text { controls } \\
\text { II }\end{array}$ \\
\hline
\end{tabular}

\begin{tabular}{|c|c|c|c|c|c|c|c|}
\hline Subjects $\mathrm{n}$ & 78 & 29 & 28 & 21 & 25 & 300 & 300 \\
\hline $\begin{array}{l}\text { Type of } \\
\text { subjects }\end{array}$ & Asbestosis & Asbestosis & Asbestosis & Asbestosis & Pleural plaques & Controls & Controls \\
\hline Origin & $\begin{array}{l}\text { Dutch/ } \\
\text { French }\end{array}$ & Dutch & Dutch & French & Dutch & Dutch & French \\
\hline $\mathbf{G G}^{\#} \mathrm{n}$ & 36 & 11 & 15 & 10 & 18 & 239 & 242 \\
\hline $\mathbf{G T}^{\#} \mathrm{n}$ & 38 & 17 & 11 & 10 & 7 & 58 & 51 \\
\hline $\mathrm{TT}^{\#} \mathrm{n}$ & 4 & 1 & 2 & 1 & 0 & 3 & 7 \\
\hline p-value & $5.1 \times 10^{-11}$ & $1.3 \times 10^{-6}$ & $3.9 \times 10^{-4}$ & $6.2 \times 10^{-4}$ & 0.5 & NA & NA \\
\hline
\end{tabular}

MAF: minor allele frequency; NA: not available. " : genotype of MUC5B rs35705950, T is minor allele. Genotype results of the discovery cohort, replication cohort I and pleural plaque cohort were compared with healthy controls I and replication cohort II with healthy controls II. The pooled cohort was compared with genotype results of healthy controls I and II combined. 
treatment decelerates disease progression [13]. This treatment option is not yet available for patients with asbestosis. However, the recently published INBUILD trial showed promising results of nintedanib in non-IPF ILD, including patients with exposure-related ILD [14]. A Dutch multi-centre trial of pirfenidone treatment in patients with asbestosis is currently being conducted (EudraCT number: 2018-001781-41).

A limitation of our study is the sample size, which is relatively small compared to numbers in genetic studies with other ILDs. Nonetheless, each independent cohort was significantly powered ( $>80 \%)$ to detect a genetic association in the range of that of IPF cohorts for the MUC5B risk allele.

In summary, we found a significant association between the minor allele of MUC5B rs35705950 and asbestosis. This adds to accumulating evidence for a MUC5B-driven endotype of pulmonary fibrosis in ILD.

Mark G.J.P. Platenburg ${ }^{1}$, Ivo A. Wiertz ${ }^{1}$, Joanne J. van der Vis ${ }^{1,2}$, Bruno Crestani ${ }^{3,4}$, Raphael Borie $\oplus^{3,4}$, Philippe Dieude $\oplus^{4,5,6}$, Caroline Kannengiesser $\oplus^{4,6,7}$, Jacobus A. Burgers ${ }^{8}$, Jan C. Grutters ${ }^{1,9}$ and Coline H.M. van Moorsel $^{1,9}$

${ }^{1}$ Interstitial Lung Diseases Center of Excellence, Dept of Pulmonology, St. Antonius Hospital, Nieuwegein, The Netherlands. ${ }^{2}$ Dept of Clinical Chemistry, ILD Center of Excellence, St Antonius Hospital, Nieuwegein, The Netherlands. ${ }^{3}$ Service de Pneumologie A, Hôpital Bichat, AP-HP, DHU FIRE, Paris, France. ${ }^{4}$ Unité 1152, INSERM, Université Paris Diderot, Paris, France. ${ }^{5}$ Service de Rhumatologie, Hôpital Bichat, AP-HP, Paris, France. ${ }^{6}$ Université de Paris, Université Paris Diderot, Paris, France. ${ }^{7}$ Laboratoire de Génétique, Hôpital Bichat, AP-HP, Paris, France. ${ }^{8}$ Dept of Thoracic Oncology, The Netherlands Cancer Institute, Amsterdam, The Netherlands. ${ }^{9}$ Division of Heart and Lungs, University Medical Center, Utrecht, The Netherlands.

Correspondence: Mark Platenburg, Dept of Pulmonology, St Antonius Hospital Nieuwegein, Koekoekslaan 1, 3435 CM Nieuwegein, The Netherlands. E-mail: m.platenburg@antoniusziekenhuis.nl

Received: 2 Oct 2019 | Accepted after revision: 5 Jan 2020

Support statement: Funding was received from ZonMw TopZorg (grant number: 842002003) and the Machiel van der Woude Stipendium from the Institute of Asbestos Victims located in the Hague, the Netherlands. Funding information for this article has been deposited with the Crossref Funder Registry.

Conflict of interest: M.G.J.P. Platenburg has nothing to disclose. I.A. Wiertz has nothing to disclose. J.J. van der Vis has nothing to disclose. B. Crestani has nothing to disclose. R. Borie reports grants and personal fees from Roche and Boehringer Ingelheim, outside the submitted work. P. Dieude has nothing to disclose. C. Kannengiesser has nothing to disclose. J.A. Burgers reports grants from MSD, institutional fees for data monitoring committee work from Roche, institutional fees for advisory board work from AstraZeneca and Boehringer Ingelheim, outside the submitted work. J.C. Grutters has nothing to disclose. C.H.M. van Moorsel has nothing to disclose.

\section{References}

1 American Thoracic Society. Diagnosis and initial management of nonmalignant diseases related to asbestos. Am J Respir Crit Care Med 2004; 170: 691-715.

2 Roth GA, Abate D, Abate KH, et al. Global, regional, and national age-sex-specific mortality for 282 causes of death in 195 countries and territories, 1980-2017: a systematic analysis for the Global Burden of Disease Study 2017. Lancet 2018; 392: 1736-1788.

3 Markowitz SB, Levin SM, Miller A, et al. Asbestos, asbestosis, smoking, and lung cancer: new findings from the North American insulator cohort. Am J Respir Crit Care Med 2013; 188: 90-96.

4 Raghu G, Collard HR, Egan JJ, et al. An Official ATS/ERS/JRS/ALAT Statement: idiopathic pulmonary fibrosis: evidence-based guidelines for diagnosis and management. Am J Respir Crit Care Med 2011; 183: 788-824.

5 Seibold MA, Wise AL, Speer MC, et al. A common MUC5B promoter polymorphism and pulmonary fibrosis. N Engl J Med 2011; 364: 1503-1512.

6 Paris C, Thierry S, Brochard P, et al. Pleural plaques and asbestosis: dose- and time-relationships based on HRCT data. Eur Respir J 2009; 34: 72-79.

7 Van Der Vis JJ, Snetselaar R, Kazemier KM, et al. Effect of $M u c 5 b$ promoter polymorphism on disease predisposition and survival in idiopathic interstitial pneumonias. Respirology 2016; 21: 712-717.

8 Juge PA, Lee LS, Ebstein E, et al. MUC5B promoter variant and rheumatoid arthritis with interstitial lung disease. N Engl J Med 2018; 379: 2209-2219.

9 Burman A, Tanjore H, Blackwell TS. Endoplasmic reticulum stress in pulmonary fibrosis. Matrix Biol 2018; 68-69: 355-365.

10 Kamp DW, Liu G, Cheresh P, et al. Asbestos-induced alveolar epithelial cell apoptosis: The role of endoplasmic reticulum stress response. Am J Respir Cell Mol Biol 2013; 49: 892-901.

11 Martinez FJ, Raghu G, Schwarz M, et al. Prednisone, azathioprine, and N-acetylcysteine for pulmonary fibrosis. N Engl J Med 2012; 366: 1968-1977.

12 Wiertz IA, Wuyts WA, van Moorsel CHM, et al. Unfavourable outcome of glucocorticoid treatment in suspected idiopathic pulmonary fibrosis. Respirology 2018; 23: 311-317.

13 Raghu G, Rochwerg B, Zhang Y, et al. An official ATS/ERS/JRS/ALAT clinical practice guideline: treatment of idiopathic pulmonary fibrosis. Am J Respir Crit Care Med 2015; 192: e3-e19.

14 Flaherty KR, Wells AU, Cottin V, et al. Nintedanib in progressive fibrosing interstitial lung diseases. $N$ Engl J Med 2019; 381: 1718-1727. 